\title{
International Journal of Medicine
}

\section{Reviewing neurosurgical management of brain metastases : Three case reports}

\author{
V.K.S Gautam ${ }^{1 *}$, Ravinder Singh ${ }^{2}$, Sarbjeet Khurana ${ }^{3}$, Ankur Shrivastava ${ }^{4}$ \\ ${ }^{1}$ Assistant Professor, Department of Neurosurgery \\ ${ }^{2}$ Associate Professor \& Head, Department of Medical Anthropology \\ ${ }^{3}$ Associate Professor \& Head, Department of Epidemiology \\ ${ }^{4}$ Senior Resident, Department of Neurosurgery Institute of Human behavior and Allied Sciences Hospital, \\ Faculty of Medical sciences, University of Delhi, India \\ *Corresponding author E-mail: drvksg@gmail.com
}

\begin{abstract}
Introduction: Brain metastasis is a major cause of morbidity and mortality in patients with systemic malignancies. Newer imaging and treatment modalities have improved clinical outcome in last few decades.

Objective: It reviews recent advances in the field of epidemiology, neuroimaging, neurosurgery, radiation therapy and other interventions affecting the clinical outcome of a patient with brain metastasis.

Materials and Methods: Case record files of 3 patients operated by the author were reviewed for analyzing clinical and radiological presentations and outcome following neurosurgical intervention. Pre and post-operative clinical evaluation, neuroimaging, neurosurgical resection and histopathological confirmation of the metastasis followed by chemoradiation therapy were done in all cases.

Results: Headache, seizures and neurological deficit were common presentations of patients with brain metastases. MRI brain with Gadolinium enhancement and MR spectroscopy were the investigations of choice. All three patients underwent neurosurgery for tumor decompression and histopathological confirmation of metastasis. Neurosurgery followed by chemoradiation therapy was beneficial in all cases.

Conclusion: The course of clinical management of a patient with brain metastasis is guided by the prognostic indicators. About five decades ago, steroids, cerebral decongestants and whole brain radiotherapy (WBRT) was the mainstay of the treatment of brain metastasis. Newer treatment options include brachytherapy, chemotherapy, stereotactic radiosurgery (SRS), stereotactic radiotherapy (SRT), stereotactic biopsy and neurosurgical excision. Aims of the neurosurgical excision are reduction of the intracranial pressure, improvement in neurological status and histopathological confirmation of the diagnosis.
\end{abstract}

Keywords: Brain Metastasi; Neurosurgical Resection; Stereotactic Radiosurgery.

\section{Introduction}

Brain metastases are the most common brain tumors. Its incidence is rising due to early detection, treatment of systemic malignancy and longer life span (Greenberg, 2010; Tandon, Ramamurthy and Jain, 2014). In view of recent advances in neuroimaging, chemoradiation therapy and availability of safe and effective therapeutic modalities, it is pertinent to evolve an evidence based guideline for the multidisciplinary management of a patient with brain metastasis.

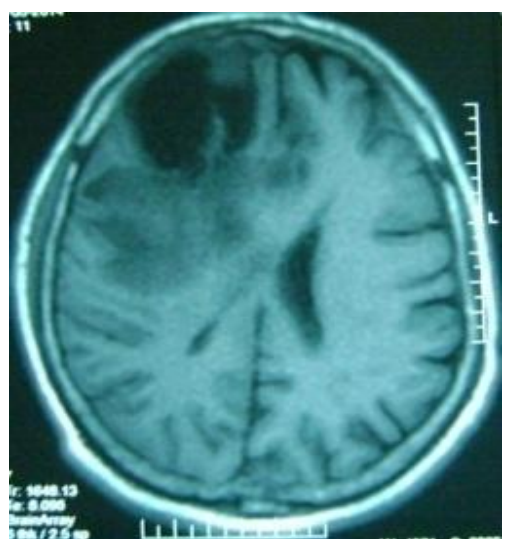

Fig. 1: T1W1 axial view of the brain showing hypo intense lesion in right frontal lobe. 


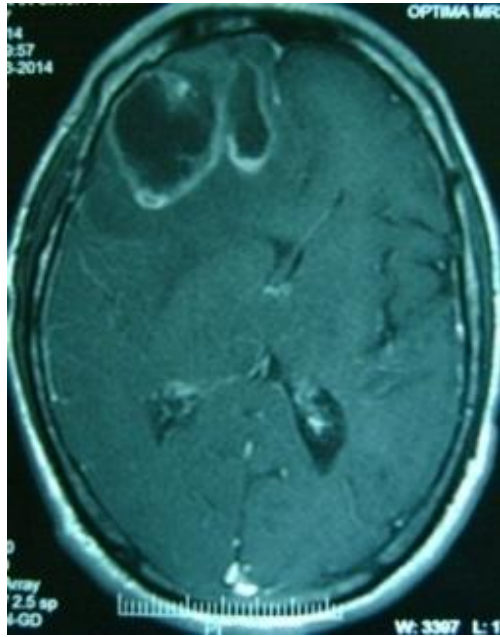

Fig. 2: T1W1 contrast enhanced (CE) axial view of the brain showing enhancing lesion in right frontal fobe.

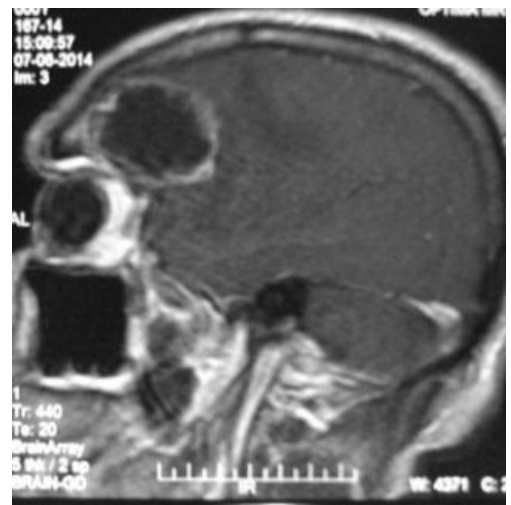

Fig. 3: T1W1 CE sagittal view of the brain reveals frontal lobe lesion.

\section{Method}

Case study method used:

Case 1: 62 year old male patient presented with history of headache, irrelevant talking (behavior changes) and forgetfulness of 2 months duration. He was a known smoker and had hypertension. Contrast enhanced CT scan and MRI brain and MR spectroscopy were suggestive of right frontal lobe metastasis without any evidence of cancer elsewhere in the body. Right frontal craniotomy and tumor decompression was done under general anesthesia. Peroperative frozen section biopsy and histopathology of the resected lesion confirmed metastasis. Patient received chemotherapy and radiotherapy during follow up period.

Case 2: 45 year old male, presented with complaints of headache vertigo, imbalance and difficulty and speech difficulty of 3 days duration. On examination cerebellar signs were present. He was a chronic smoker and alcoholic. Chest radiograph was suggestive of primary cancer of the lung. MRI brain revealed cerebellar lesion of variegated intensity with peripheral rim enhancement with features suggestive of posterior fossa space occupying lesion with hemorrhage and necrosis. Another small lesion was also present in the left cerebellar hemisphere with perilesional edema, obstructive hydrocephalus with tonsillar herniation. Provisional diagnosis of left cerebellar metastasis was made and left suboccipital craniectomy was done in prone position under general anesthesia. Histopathology of the resected tissue showed papillary adenocarcinoma.

Case 3: 58 year old male patient admitted with history of dizziness for 3 months duration and one episode of seizure about 6 months back. He was a known smoker and hypertensive. He also had features of gastritis for duration of about 25 years. Ultrasonogram (USG) of the abdomen was normal. Antiepileptic, antihypertensive medications and cerebral decongestant were pre- scribed. MRI of the brain with contrast revealed a well marginated lesion with central necrosis and thick enhancing nodular solid ring in left parieto occipital region. Chest X-Ray postero-anterior view showed an ill-defined opacity in right lower zone of the lung. Chest CT scan with contrast revealed a well marginated contrast enhancing soft tissue density lesion involving right middle lobe with an osteolytic expansile lesion at D12 vertebral on left side suggestive of mitotic pathology. Left parito-occipital craniotomy and tumor decompression was done. Histopathological examination of the resected lesion established the diagnosis of metastatic papillary adenocarcinoma.

The present study was done in the Institute, a tertiary care neuropsychiatry hospital in Delhi, India. We have analyzed three patients of brain metastases and have reported in the paper. The author had managed these patients in the Neurosurgical unit of the Institute between March 2009 and July 2014.

All the patients were investigated systematically through various diagnostic tests. Clinical and neuroradiological review of all patients either with CT scan or MRI of the brain or both were suggestive of brain metastasis. Prior to surgery all patients were given cerebral decongestants like Glycerol, Acetazolamide and corticosteroid. Neurosurgical procedure was done as per the required intervention according to the neurosurgical diagnosis of the patient and the evidence based practice. Per-operative frozen section biopsy was obtained in all cases. Frozen section biopsy and histopathological examination of the resected tumor tissue confirmed the diagnosis of brain metastasis in all cases. All cases were given chemoradiation therapy and further investigations were advised in follow up period for evaluation of any clinical deterioration or recurrence of the tumor.

\section{Result}

The common presentation of brain metastasis was headache. Craniotomy and tumor decompression was the common modality of neurosurgical intervention. In Case-2, a case of cerebellar metastasis, suboccipital craniectomy and tumor resection was done. For pre-operative diagnosis MRI brain with contrast with MR spectroscopy was the most appropriate modality of investigation. In all cases per-operative frozen section biopsy and histopathological examination of the resected tumor tissue confirmed the diagnosis of brain metastasis. All three cases exhibited significant neurological improvement following surgery and chemoradiation therapy.

\section{Discussion}

In last 50 years, there has been nearly a five-fold increase in the overall prevalence of brain metastases; the ratio between metastatic to primary brain tumor is now almost 50:50 (Osborn, 2013). The incidence of metastases is rising due to early detection by advanced neuroimaging modalities and effective treatment regimens of systemic malignancies (Eichler and Loeffler, 2007).

Up to $30 \%$ of patients with cancer develop cerebral metastasis (Mintz et al, 2006). Autopsy studies of patients who die of cancer revealed that CNS metastases occur in about $25 \%$ of patients (Wesseling, Deimling, Aldape, 2007). In patients with no history of cancer a cerebral metastasis was the presenting symptom in $15 \%$ (Soffietti, et al, 2006). Highest incidence of brain metastases is seen in $5^{\text {th }}$ to $7^{\text {th }}$ decade of life (Lang et al, 2011). In our study, the age of patients ranges from 45 to 62 years.

Metastases most commonly spread via through hematogenous route (Osborn, 2013). The brain parenchyma is the most common site $(80 \%)$, followed by the skull and dura (15\%) (Eichler and Loeffler, 2007; Khosla and Sharma, 2007). Direct extension to brain from a cancer of adjacent structures like cancers of nasopharynx, paranasal sinuses, middle ear (e.g. squamous cell carcinoma, esthenioneuroblastoma) is much less common than hematogenous spread (Osborn, 2013, Wesseling, Deimling, Aldape, 2007). Diffuse leptomeningeal (pial) and subarachnoid space infiltrations are relatively uncommon, accounting for just 
5\% of all cases (Osborn, 2013). Brain metastases are preferably located in arterial border zones and at the junction of cerebral cortex and subcortical white matter (Wesseling, Deimling, Aldape 2007, Chi and Komaki 2010). About 15\% of metastases are found in the cerebellum. The midbrain, pons and medulla oblongata are uncommon sites and account for less than $1 \%$ of metastases (Osborn, 2013). Other rare sites include the choroid plexus, ventricular ependyma, pituitary gland and retinal choroid (Osborn, 2013, Wesseling, Deimling, Aldape, 2007). The metastasis may also occur through the CSF pathway and may present as drop metastasis. Primary brain tumors like germinoma and medulloblastoma may spread along CSF pathways (Wesseling, Deimling, Aldape, 2007). Some systemic cancer like lymphomas and leukemias involve leptomeninges and is known as meningeal carcinomatosis. It is a diffuse metastasis in the leptomeninges by carcinomatous infiltration (Wesseling, Deimling, Aldape, 2007). In this study all three cases revealed parenchymal brain metastasis where spread occurred through hemtogenous route.

Single metastasis accounts for one third to one quarter of patients with brain metastasis (Norden, et al, 2005). About $20 \%$ of patients have two lesions, $30 \%$ have three or more and only $5 \%$ have more than 5 lesions (Osborn, 2013).

The most common sources of brain metastases in adults are, in descending order, lung cancer (especially small cell and adenocarcinoma), breast cancer, melanoma, renal carcinoma and colon cancer (Lang et al, 2011). In children in descending order of frequency, they are leukemia, lymphoma and sarcoma (Wesseling, Deimling, Aldape, 2007). Melanoma, although constitute only 4\% of all cancers, has the highest propensity to result in brain metastasis (Eichler and Loeffler, 2007, Lang et al, 2011, Siu and Lang, 2012).

The average period required for the development of brain metastasis from lung cancer is 4 to 10 months, whereas it is approximately 3 years in breast cancer (Siu and Lang, 2012). We found in our study the duration of illness varied from 3 days to 6 months. Histopathology of the lesion usually reflects the tissue of origin, i.e. the primary site of cancer. The histological features are as diverse as in the primary tumors from which they arise. Metastatic choriocarcinoma should be considered in the differential diagnosis of hemorrhagic intracranial masses in females of child bearing age and surgically resected blood clot should be examined histologically for determining the etiology (Wesseling, Deimling, Aldape, 2007).

As with other intracranial space occupying lesions the symptoms and signs depend on the size and site of the lesion, raised intracranial pressure, hemorrhage, meningeal irritation and hydrocephalus. Headache, seizures and focal neurologic deficits are the most common presenting symptoms of parenchymal metastases (Eichler and Loeffler, 2007). Detailed neuropsychological testing demonstrates cognitive impairment in $65 \%$ of patients with brain metastsasis (Eichler and Loeffler, 2007).

Preoperative metastatic work up includes detailed history and systemic examination to detect the primary cancer, metastases elsewhere in the body and to rule out other diagnosis like brain abscess, tuberculosis, toxoplasmosis, neurocysticercosis, resolving hematoma, lymphoma, hemangioblastoma and glioblastoma.

Chest X-ray, radiograph of the spine, ultrasound of abdomen and pelvis, trans rectal ultrasound, mammography, bronchoscopy, upper GI and lower GI endoscopy, bone marrow examination, radionuclide bone scan, serum electrophoresis, intravenous pyelogram (IVP), CT scan of the brain, chest, abdomen and pelvis, positron emission tomography ( PET)-CT or PET-MRI may be required for detection of the systemic cancer. (Joseph, Shehadi and Seaman, 2010). Other important investigations include erythrocyte sedimentation rate (ESR), $\mathrm{C}$ - reactive protein (CRP) as markers of infection, Western Blot Test for HIV status, Gram stain, and blood and urine culture to identify hematogenous origin to an abscess. Few investigations are very costly and associated with risks and are required in very rare circumstances.

After a detailed clinical and radiological work up, systemic malignancies were detected in two cases besides brain metastasis. In one case, there was no evidence of systemic cancer elsewhere in the body.

CT scan and MR are the most commonly used techniques for detecting brain metastases. Brain metastasis appears discrete ring or disc like at subcortical location, at the junction of grey and white matter, with enhancement and extensive surrounding edema which is disproportionate to the size of the lesion. FLAIR image, contrast image, magnetization transfer (MT), MR angiography (MRA), Diffusion weighted imaging (DWI), fat suppression, MR spectroscopy further enhance the value of MRI as the investigation of choice for detecting CNS metastasis (Joseph, Shehadi and Seaman, 2010)

In carcinomatous meningitis MRI may reveal nodular contrast enhanced lining along the CSF pathways with or without hydrocephalus. FLAIR sequence is of particular value as it may reveal the neoplastic spread along the spinal cord and spinal nerves.

Prominent lipid signal is the dominating peak on MRS in the majority of brain metastases. However, lipid is also common in cellular processes including inflammation and necrosis. Choline is generally elevated, and Creatinine is depressed or absent in most metastases. CSF examination may reveal carcinomatous cells. Meningeal biopsy is indicated when imaging fails to support the diagnosis but this disease is strongly suspected.

About $11 \%$ patients with known primary cancer do not have metastatic lesion, despite the fact that CT scan or MRI suggest so. Half of these patients can have potentially curable inflammatory and so a histopathological confirmation must be obtained before planning treatment. Whole body PET-CT scanner can detect any significant residual or recurrent FDG avid lesion at the primary cancer site, or status of the lymph nodes, lungs, liver, spleen, kidneys, urinary bladder and other organs and systems like skeletal system. PET is used to evaluate the therapy response and to assess the disease status. However, PET does not distinguish secondary from primary neoplasms and may be false positive in some benign lesions of the brain.

Therapeutic approaches include steroid therapy, stereotactic biopsy, neurosurgical resection, brachytherapy, SRS, WBRT, chemotherapy and combinations of treatment (Chi and Komaki, 2010, Nader and Sabbagh, 2010, Siu, and Lang, 2012). Patient selection for a particular type of treatment is paramount in order to maximize survival and neurologic function whilst avoiding unnecessary treatment. Clinical and radiographic prognostic factors and histology of the lesion are the most important determinants of the outcome. Good prognostic indicators include Karnofsky performance status (KPS) of more than 70, age less than 65 and controlled primary tumor and no extracranial metastasis (Chi and Komaki, 2010; Norden, Wen and Kesari, 2005, Eichler and Loeffler, 2007, Gaspar et al 1997). Other prognostic factors include the sensitivity of the tumor to therapy and number and location of CNS metastases.

Anti-edema measures like Frusemide, Mannitol, Glycerol, Acetazolamide and Dexamethasone reduce the raised ICP. Steroids have an oncolytic effect and cause shrinkage of metastatic lymphomas. The mainstay of treatment for brain metastsasis over the past 5 decades has been corticosteroids and WBRT. Non-randomized studies suggest that WBRT increases the median survival time by 3-4 months over approximately 1 month without treatment and 2 months with corticosteroids (Eichler and Loeffler, 2007, Chi and Komaki, 2010, Siu and Lang, 2012).

Radiotherapy can be delivered by fractionated external beam irradiation, small field streotactic irradiation (stereotactic radiotherapy) or interstitial implantation (brachytherapy) (Dagnew et al, 2007, Patchell et al 1998). For whole brain radiation therapy (WBRT), the most common regimen employed is 35 Gy delivered in 2.5 Gy fractions over 14 treatment days. Daily fraction of more than 3 Gy likely increases the risk of neurotoxicity.Stereotactic radiotherapy (SRT) is delivered using a linear accelerator. A fractionated schedule is followed maintaining the targeting technique of SRS (Ramamurthy and Harinivas, 2014).

For neoplastic spread to the spinal cord, treatment involves the irradiation of the entire neuraxis with chemotherapy including 
methotrxate, cytosine arabinoside, and thiotepa. Intra-CSF drug therapy can also also be given. Topotecan, an inhibitor of totpisomerase-1, crosses the blood brain barrier (BBB) and may be effective in treatment of brain metastasis from small cell lung and breast cancer (Norden, Wen and Kesari, 2005; Wong and Berkenbit, 2004). Temozolamide, an oral alkylating agent also crosses BBB is useful in treating brain metastasis (Norden, Wen and Kesari, 2005).

Stereotactic radiosurgery (SRS) is a relatively recent therapeutic option that has significantly improved the effectiveness of and morbidity associated with radiation therapy. SRS may use gamma knife (GK) or linear accelerator (LINAC, CyberKnife) delivers a single large dose of focused radiation to lesions localized by stereotaxy. SRS is useful for lesions less than $3 \mathrm{~cm}$ to $4 \mathrm{~cm}$ where a radiation of 1,600-3,500 cGy is delivered in a single sitting. A major advantage of this technique over conventional surgery is that it can treat surgically inaccessible tumors in the eloquent area of the brain. SRS is a safe alternative to surgical excision in elderly frail patients with associated medical conditions, such as diabetes mellitus, hypertension (Ramamurthy and Harinivas, 2014, Chi and Komaki, 2010). SRS is a non-invasive procedure and it obviates the need of multiple craniotomies in a patient with multiple metastases. Some tumors are very sensitive to radiation like metastasis from lymphoma, germ cell tumor and small cell lung cancer where SRS is the treatment of choice.

Surgical resection is the treatment of choice in all the patients where lesion is surgically accessible, associated with significant mass effect and hydrocephalus. If the lesion is more than $4 \mathrm{~cm}$ in size, or highly cystic then surgical intervention is definitely better than SRS. If a patient presents with mass effect, local irritation of the adjoining brain tissue, raised intracranial pressure and tumor is in surgically accessible area of the brain then immediate tumor excision or decompression should be done. If the primary cancer is controlled and the life expectancy is more than 3 months, the surgery is indicated. Surgery is life saving and helps in establishing the histopathological diagnosis. The importance of diagnosis is paramount when the diagnosis of brain metastasis is in question. This is important because as many as $10 \%-15 \%$ of patients with a clinical diagnosis of metastasis may actually have non-metastatic lesions such as abscess (Wesseling, Deimling, Aldape, 2007, Lang et al, 2011).

Surgical excision should be considered for patients with good KPS score, minimal or no evidence of extracranial disease and surgically accessible brain metastasis. Surgery is needed in cancers which are resistant to radiation like thyroid carcinoma, renal cell carcinoma and melanoma. A certain, immediate and predictable outcome of surgical resection and long term local control metastatic lesion are major advantages over radiation based treatment modalities (Mintz, et al 2006, Siu, and Lang, 2012). Stereotactic biopsy should be an option for lesions located in inaccessible and eloquent area of the brain (Mintz, et al 2006).

After surgery or SRS, adjuvant WBRT is recommended. It is an effort to erradicate residual cancer cells at the resected site and to eliminate microscopic foci at distant sites within the brain, thereby, reducing the risk of tumor recurrence (Mintz, et al 2006, Siu, and Lang, 2012).Long term follow up of the patient is mandatory for evaluation of neurological status, complications of chemoradiation therapy, detection of recurrence or appearance of any new lesion, neurocognitive impairment and for neurorehabilitation and supportive care.

\section{Conclusion}

In a suspected case of brain metastasis choice of investigation, neuroimaging and therapy must be decided by the treating team based on a firm understanding of the prognostic indicators and other parameters. Patient selection is the cornerstone of management with brain metastasis. Prompt decision and aggressive management with combined modality of treatment minimizes cost of care, maximizes the clinical outcome and reduces the mortality and morbidity of patients with brain metastases.

\section{References}

[1] Greenberg M S. Cerbral metastasis. In: Handbook of Neurosurgery, $7^{\text {th }}$ ed. , Thieme; 2010, p. 702-710

[2] Ramamurthy R, Harinivas. Metastatic Brain tumors. In:.Tandon PN, Ramamurthy R, Jain P K N. Ramamurthy \& Tandon's Manual of Neurosurgery (ed), 1st ed, 2014, p. 1049-1064

[3] Osborn A G. Metastases and paraneoplastic syndrome.In: Osborn Brain imaging, Pathology and Anatomy, $2^{\text {nd }}$ ed., Amirsys; 2013, p. 745-771

[4] Eichler A.F. and Loeffler J.S. Multidisciplinary management of brain metastases. The Oncologist, 2007; 12: 884-898 http://dx.doi.org/10.1634/theoncologist.12-7-884.

[5] Mintz, A.; J.Perry, K. Spinthoff, A. Chambers and N.Laperriere. Management of single brain metastasis: a practice guideline. Current Oncology, 2006,Volume 14, number 4,130-143

[6] Wesseling P, Deimling A von, Aldape K D. Metastatic tumors of the CNS. In: WHO Classification of Tumors of the Central Nervous System, Louis D N, Olgaki H, Weistler O D, and Cavenee W K. ed., 2007, p 247-251

[7] Soffietti R, Cornu P, Delattre J Y, et al. EFNS guidelines on diagnosis and treatment of brain metastasis: Report of an EFNS task force. European journal of Neurology, 2006, 13; 674-681. http://dx.doi.org/10.1111/j.1468-1331.2006.01506.x.

[8] Lang F F, Chang E L, Suki D, Wildrick D M, Sawaya R. Metastatic brain tumors. In: Winn H R, Youmans Neurological Surgery, $6^{\text {th }}$ Edition, Vol 2 (Elsevier Saunders), 2011, p 1410-1425

[9] Khosla V K, Sharma B S. Surgical management of brain metastasis. In: Textbook of Operative Neurosurgery. Ramamurthy R, Sridhar K, Vasudevan M C., ed., 2007, B.I. Publication Pvt Ltd, New Delhi, p 545-551

[10]Chi A, Komaki R. Treatment of brain metastasis from lung cancer, Cancer2010, 2, 2100-2137. http://dx.doi.org/10.3390/cancers2042100.

[11]Norden A D, Patrick Y, Wen and Kesari S. Brain metastases. Current Opinion in Neurology, 2005, 18; 654-661.

[12] Joseph A, Shehadi and Seaman B. Single brain metastases. In: Neurosurgery Case Review: Questions and Answers, ed., Nader R, Sabbagh A J, Thieme, 2010, p 58-61

[13]Siu T, Lang F F. Surgical management of cerebral metastasis. In: Schmidek \& Sweet operative Neurosurgical techniques, indications, methods and results, Alfredo Quinones- Hinojosa, $6^{\text {th }}$ edition, ( Elsevier Saunders), 2012, p 178-91

[14]Gaspar L, Scott C, Rotman M, et al. Recursive partitioning analysis (RPA) of prognostic factors in three Radiation Therapy Oncology Group (RTOG) brain metastases trials. International Journal of Radiation Oncology, Biology, Physics 1997; 37: 745-751. http://dx.doi.org/10.1016/S0360-3016 (96)00619-0.

[15] Wong ET, Berkenbit A. The role of topotecan in the treatment of brain metastasis.Oncologist, 2004;9:68-79. http://dx.doi.org/10.1634/theoncologist.9-1-68

[16]Dagnew E, Kanski J, Mc Dermott MW, et al. Management of newly diagnosed single brain metastasis using resection and permanent iodine-125 seeds without initial whole-brain radiotherapy: a two institution experience. Neurosurg Focus 2007; 22(3):E3 http://dx.doi.org/10.3171/foc.2007.22.3.4.

[17]Patchell RA, Tibbs PA, Regine WF, et al. Postoperative radiotherapy on the treatment of single metastasis to the brain: a randomized trial. JAMA, 1998;280:1485-1489. http://dx.doi.org/10.1001/jama.280.17.1485 